\title{
EDITORIAL
}

\section{A special issue on Optoelectronics in Energy}

\author{
Changqing CHEN (凶), Jun ZHOU (ه), Yanyan FANG (凶) \\ Wuhan National Laboratory for Optoelectronics, Huazhong University of Science and Technology, Wuhan 430074, China
}

(C) Higher Education Press and Springer-Verlag Berlin Heidelberg 2013

With the gradual reduction of fossil fuel and the growing environmental concerns over the climate change associated with the use of fossil fuel, renewable energy sources such as solar, wind, biomass, geothermal and hydroelectric are becoming increasingly important. In particular, solar power generation has emerged as the most rapidly growing renewable source. As a result, solar cells of different types have been intensively studied. In this special issue, 2 review articles and 2 research articles focusing on solar cells are presented. Dye-sensitized solar cells (DSSCs) are regarded as one of the most promising types due to their low-cost, transparency and relatively high conversion efficiency. Dr. Hongwei Han et al. reviewed the recent progress of materials and achievements for all-solid-state DSSCs and highlighted some representative examples. Also, in a research article, they reported a monobasal solidstate DSSCs with mesoporous $\mathrm{TiO}_{2}$ beads, and as high as $4 \%$ efficiency is achieved under air mass (AM) 1.5 illumination. Dr. Mingkui Wang et al. introduced the design and understanding of sensitizers, which are extremely important in determining the performance of DSSCs. The advances in the conception and performance of various sensitizers including ruthenium complexes, organic dyes and porphyrins are discussed. Dr. Guoli Tu et al. synthesized five 4,7-dithien-2-yl-2,1,3-benzothiadiazole (DTBT)-based conjugated copolymers with controlled molecular weight. Compared with the fluorene-based polymer, the carbazole-DTBT copolymer showed higher short circuit current density $\left(J_{\mathrm{sc}}\right)$ and power conversion efficiency (PCE) value that was due to its better intermolecular stacking.

In addition to seeking the renewable energy sources, people also have been engaging in a variety of energysaving technologies to reduce the consumption of fossil fuel, among which the technology of light-emitting diodes (LEDs) plays an important role. In this special issue, we selected 2 research articles focusing on LEDs. Dr. Xiong Hui et al. theoretically studied the effects of gradually increased barrier heights from $n$ - to $p$-layers in the active region on blue InGaN based LEDs. And Dr. Lei Wang et al. demonstrated a highly efficient phosphorescent organic lighting emitting diodes (PhOLEDs) with low efficiency roll-off by using a unilateral homogenous device structure with wide band-gap material 4,4',4"-tri( $\mathrm{N}$-carbazolyl)-triphenylamine (TCTA) as hole transporting layer and emitting layer (EML).

This special issue also features 1 review article and 3 research articles focusing on nano-materials. Dr. Xiuquan Gu et al. summarized the growth mechanisms and the recent progresses of $\mathrm{ZnO}$ nanostructures for the application of electron field emitters. Dr. Yu Tian reported a simple and effective method to fabricate highly ordered $\mathrm{ZnO}$ nanorod arrays on $\mathrm{H}_{2}$-decomposed $\mathrm{GaN}$ epilayer via hydrothermal route. Dr. Qing Yang investigated the lasing characteristics of curved semiconductor nanowires and found the abnormal phenomenon of dominant peak switching when increasing the pump power. Dr. Chaojian Wu synthesized hydrophilic photoluminescent CdTe/poly (1, 4-butanediol-citrate) (PBC) bioelastomer nanocomposites by a two-step method and found these nanocomposites possess good hydrophilicity and high fluorescence properties.

Additionally, Dr. Mingqiang Zhu reported the synthesis of hexaarylbiimidazole-tetraphenylethene (HABI-TPE) conjugated photochromic fluorophore, which simultaneously exhibited photochromic property, condensed state enhanced emission and reversible fluorescence switching. And Dr. Yiwei Xie proposed and demonstrated an allfiber tunable and programmable bandpass filter using a linearly chirped fiber Bragg grating (CFBG).

Received November 5, 2013

E-mail: cqchen@mail.hust.edu.cn, jun.zhou@mail.hust.edu.cn, yanyan.fang@hust.edu.cn 
We would like to thank to all of the authors for their excellent contributions and the editors of Frontiers of Optoelectronics for inviting us to act as guest editors for this special issue.

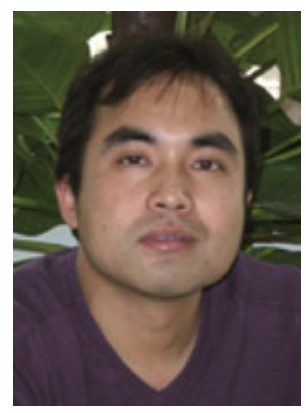

Changqing Chen, as a scholarship holder of DAAD Volkswagen Foundation, achieved his Ph.D degree in semiconductor materials and devices from University of Erlangen-Nürnberg, Germany in 2000. During 2001 to 2004, he served as a research scientist in Virginia Commonwealth University and University of South Carolina. Dr. Chen joined Huazhong University of Science and Technology and Wuhan National Laboratory for Optoelectronics (WNLO) in Nov. 2007. His current research interest is III-nitride materials and devices including ultraviolet lightemitting diodes, ultraviolet photodetectors and AlGaN/GaN MQWs based intersubband transition infrared photodetectors.

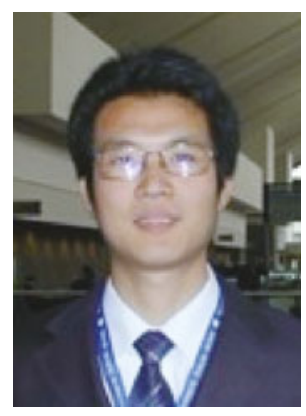

Jun Zhou obtained his Ph.D degree in materials physics and chemistry in Sun Yat-sen University in 2007. Before Dr. Zhou joined Huazhong University of Science and Technology and Wuhan National Laboratory for Optoelectronics (WNLO) in Dec. 2009, he served as a research scientist in the Wallace H. Coulter Department of Biomedical Engineering and School of Materials Science and Engineering, Georgia Institute of Technology. He received the Excellent Doctoral Dissertation Award of China in 2009. His current research interest is fabricating flexible self-powered nanosystem including all-solid-state flexible supercapacitors and flexible optoelectronic nanosensors. Since 2012, he is the assistant director of WNLO.

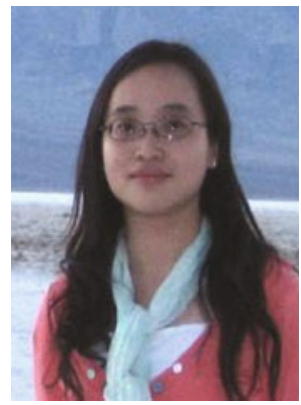

Yanyan Fang received her Ph.D degree in materials science and engineering in Arizona State University in 2009. Then she joined Huazhong University of Science and Technology and Wuhan National Laboratory for Optoelectronics (WNLO) in Dec. 2009. Her current research interest is the AlGaN based ultraviolet optoelectronic devices. 